\title{
Clinical and genetic characteristics of female dystrophinopathy carriers
}

\author{
JINGZI ZHONG $^{1 *}$, YANSHU XIE ${ }^{1 *}$, VIDATA BHANDARI $^{1}$, GANG CHEN $^{2}$, \\ YIWU DANG $^{2}$, HAIXIA LIAO ${ }^{1}$, JIAPENG ZHANG ${ }^{1}$ and DAN LAN ${ }^{1,3}$ \\ Departments of ${ }^{1}$ Pediatrics and ${ }^{2}$ Pathology; ${ }^{3}$ Team for Major Disease Prevention and Control, The First \\ Affiliated Hospital of Guangxi Medical University, Nanning, Guangxi 530021, P.R. China
}

Received May 17, 2018; Accepted February 5, 2019

DOI: $10.3892 / \mathrm{mmr} .2019 .9982$

\begin{abstract}
The present study aimed to determine the genetic status of manifesting carriers (MCs) of Duchenne muscular dystrophy (DMD)/Becker muscular dystrophy (BMD) and asymptomatic carriers with a family history of DMD/BMD, and identify potential simple and reliable methods for screening dystrophinopathy carriers. Clinical data from probable carriers and MCs were collected and analyzed. MCs underwent multiplex ligation-dependent probe amplification (MLPA) for dystrophin gene exons combined with muscle disease panel test based on a next-generation sequencing (NGS) platform. In addition, the status of probable carriers was determined by MLPA or Sanger sequencing, according to the mutations of probands. A total of 154 female were enrolled, among which 78 cases were found to be carriers, including $4 \mathrm{MCs}$ and 74 asymptomatic female carriers. The $4 \mathrm{MCs}$ exhibited duplication mutations. Among the 74 asymptomatic carriers, $41.89 \%$ harbored deletion mutations, including 2 cases with suspected germline mosaicism and no mutation in the dystrophin gene, while $44.59 \%$ harbored point mutations in exons and only 10 cases $(13.51 \%)$ carried duplication mutations. The area under the receiver operating characteristic
\end{abstract}

Correspondence to: Dr Dan Lan, Department of Pediatrics, The First Affiliated Hospital of Guangxi Medical University, 6 Shuangyong Road, Nanning, Guangxi 530021, P.R. China

E-mail: land6785@163.com

${ }^{*}$ Contributed equally

Abbreviations: ALT, alanine transaminase; AST, aspartate transaminase; BMD, Becker muscular dystrophy; CK, creatine kinase; DMD, Duchenne muscular dystrophy; InDels, insertions or deletions; LGMD, limb-girdle muscular dystrophy; NGS, next-generation sequencing; MCs, manifesting carriers; MLPA, multiplex ligation-dependent probe amplification; ROC, receiver operating characteristic; SNP, single-nucleotide polymorphism

Key words: Duchenne muscular dystrophy, Becker muscular dystrophy, manifesting carriers, asymptomatic carriers, genetic analysis, germline mosaicism
(ROC) curve of creatine kinase (CK) was 0.822 , with a sensitivity of $65.38 \%$ and specificity of $92.1 \%$. In addition, DMD was positively correlated with the CK, alanine transaminase and aspartate transaminase levels of the carriers. MLPA for exons of the dystrophin gene, along with NGS and Sanger sequencing, was effective for the diagnosis of MCs and for determining the status of probable carriers. The ROC curve analysis also demonstrated that CK level was an excellent predictor for distinguishing DMD/BMD carriers.

\section{Introduction}

Duchenne muscular dystrophy (DMD) and Becker muscular dystrophy (BMD) are the most common childhood muscular dystrophies, and are caused by mutations in the dystrophin gene, which encodes dystrophin protein at the Xp-21 locus. DMD and BMD occur with a birth prevalence rate of 15.9-19.5 cases per 100,000 live male births and 1 case per $6,000-8,000$ live male births, respectively $(1,2)$. Approximately $60-65 \%$ of mutations are deletions, $5-15 \%$ are duplications and $30 \%$ are point mutations (3). There are two hotspots in the dystrophin gene. The most important hotspots are located in exon 45-55 and exon 2-19 (4). However, DMD and BMD are $\mathrm{X}$-linked recessive diseases, and thus females are the carriers of the mutation.

The majority of DMD/BMD carriers remain asymptomatic, while only $2.5-10 \%$ of carriers are symptomatic and classified as manifesting carriers (MCs) (5). The symptoms of MCs range from mild muscle weakness to severe abnormal gait with frequent falls, and difficulty in rising from the floor or walking on tiptoes. Certain patients even have rapidly progressive muscular dystrophy similar to male dystrophinopathy. Diagnosis of DMD/BMD carriers is based on clinical symptoms, family history, biochemistry markers, echocardiography, pathology, molecular genetic analysis and linkage-testing. Identification of $\mathrm{DMD} / \mathrm{BMD}$ carriers may present challenges, particularly in the absence of a family history of DMD/BMD. Genetic analysis of the dystrophin gene is typically required for diagnosis, particularly for genetic consulting and prenatal diagnosis.

In the present study, clinical data were collected and analyzed from 154 probable female carriers. The creatine kinase (CK) level of the subjects, and its correlation with 
alanine transaminase (ALT) and aspartate transaminase (AST) levels were analyzed. Multiplex ligation-dependent probe amplification (MLPA) for exons of the dystrophin gene, combined with a muscle disease panel based on next-generation sequencing (NGS), was applied to detect the MCs. Two of the MCs were confirmed by muscle pathological examination. The status of probable carriers was determined by MLPA and Sanger sequencing according to the mutations of probands. The present study presents a non-invasive, easy screening for MCs of DMD/BMD, and describes an effective, accurate method for the detection of deletions/duplications and small mutations in DMD/BMD carriers.

\section{Materials and methods}

Participants. The study participants were selected between January 2013 and October 2017 at the First Affiliated Hospital of Guangxi Medical University (Nanning, China). The subjects included male DMD patients (proband), symptomatic females and their relatives, including mothers, maternal grandmothers, maternal aunts and sisters of probands with confirmed DMD dystrophin gene mutations. The present study was conducted in strict accordance with the recommendations in the Guidelines of the Centers for Disease Control and Prevention (1). The study was approved by the Ethics Committee of the First Affiliated Hospital of Guangxi Medical University. Informed consent was obtained from all participants included in the study.

Inclusion and exclusion criteria. Subjects included in the present study fulfilled one of the following criteria: i) Exhibited clinical manifestations, such as muscle weakness, walking with frequent falling, abnormal gait, and difficulty running, jumping and going upstairs; ii) were maternal female relatives of probands with confirmed diagnosis of DMD/BMD; and iii) exhibited a persistent $\mathrm{CK}$ level that was $>2$ times greater than the upper limit of the normal range $(\mathrm{CK},<178 \mathrm{U} / 1)$. Symptomatic females who were confirmed by genetic testing to suffer from another muscular dystrophy disease, such as limb-girdle muscular dystrophy (LGMD), or females with incomplete clinical information were excluded from the present study.

Clinical analysis. The clinical data (including the chief complaint, history of present illness, growth and development history, past medical history and family history) of all subjects were collected and reviewed retrospectively. Blood samples were collected and levels of serum enzymes CK, liver function and cardiac enzymes concentrations were measured using a Hitachi 7600-020 analyzer (Hitachi, Ltd., Tokyo, Japan). All assays were conducted according to the manufacturer's instructions using the same batch reagent. Echocardiography findings and muscle biopsies were analyzed.

Genetic analysis. All probands and MCs underwent MLPA for dystrophin gene exons. If no mutation was identified by MLPA, NGS-based muscle disease panel tests (containing 169 known muscle disease-associated genes, including for LGMD and facioscapulohumeral muscular dystrophy) was performed. When large deletions/duplications were observed by MLPA analysis, MLPA was also used to classify the female relatives. If a point mutation was identified by NGS in probands, The mutation site was amplified by PCR (the primers were designed by the website Primer Z(http://genepipe.ncgm.sinica. edu.tw/primerz/primerz4.do), and then sequenced by Sanger sequencing for probands and their female relatives.

MLPA analysis. DNA samples were extracted from peripheral blood obtained from the subjects according to standard procedures [FlexiGene DNA kit (cat. no. 51206; Qiagen GmbH, Hilden, Germany)]. All 79 exons of the dystrophin gene were screened by MLPA. Two sets of reagents (SALSA MLPA probe sets $\mathrm{P} 034$ and P035) were used to perform the MLPA reaction according to the manufacturer's instructions (MRC-Holland BV, Amsterdam, The Netherlands). Genomic DNA was denatured, hybridized, ligated, and amplified. Amplified products were analyzed on an ABI model 3500XL capillary sequencer (Thermo Fisher Scientific, Inc., Waltham, MA, USA). The initial data were analyzed using GeneMapper software (version 140701.0000), and the peak area of each fragment was compared with that of 3 control samples.

Target enrichment of genomic DNA and sequencing. A minimum of $3 \mu \mathrm{g}$ DNA was used to construct the indexed Illumina libraries according to the manufacturer's instructions. A final library size of 200-300 bp, including adapter sequences, was selected. All exons of the dystrophin gene were target-enriched using the dystrophin Exon Enrichment kit (MyGenostics, Inc., Baltimore, MD, USA). The muscle diseases panel was a complete kit designed by the Zhongguancun Huakang Gene Institute (Beijing, China) and synthesized using the Agilent SureSelect Target Enrichment technique (Agilent Technologies, Inc., Santa Clara, CA, USA). The capture experiment was conducted according to the manufacturer's instructions. The enrichment libraries were sequenced on an Illumina HiSeq 2000 sequencer (Illumina, Inc., San Diego, CA, USA) for paired-read 100 bp sequencing.

Bioinformatics analysis. Following HiSeq 2000 sequencing (Illumina, Inc.), high-quality reads were retrieved from raw reads by filtering out the low-quality reads and adaptor sequences using the Solexa QA package (sourceforge. net/projects/solexaqa/files/) and the cutadapt program (hpc. nih.gov/apps/cutadapt.html), respectively. The SOAPaligner program (soap.genomics.org.cn/) was then used to align the clean read sequences to the human reference genome (hg19). To detect exon duplications and deletions, the coverage of each position was plotted by base position. Higher coverage of a region indicated duplication, whereas regions that were not covered corresponded to deletions. Identical sequences were produced by polymerase chain reaction (PCR) duplication to obtain cluster formation (human genomic DNA template). After the PCR duplicates were removed using Picard software (http://broadinstitute.github. io/picard/; version 2.6.0-SNAPSHOT), single-nucleotide polymorphisms (SNPs) were identified using the SOAPsnp program (http://soap.genomics.org.cn/soapsnp.html). Subsequently, the reads were realigned to the reference genome using BWA, and insertions or deletions (InDels) were 


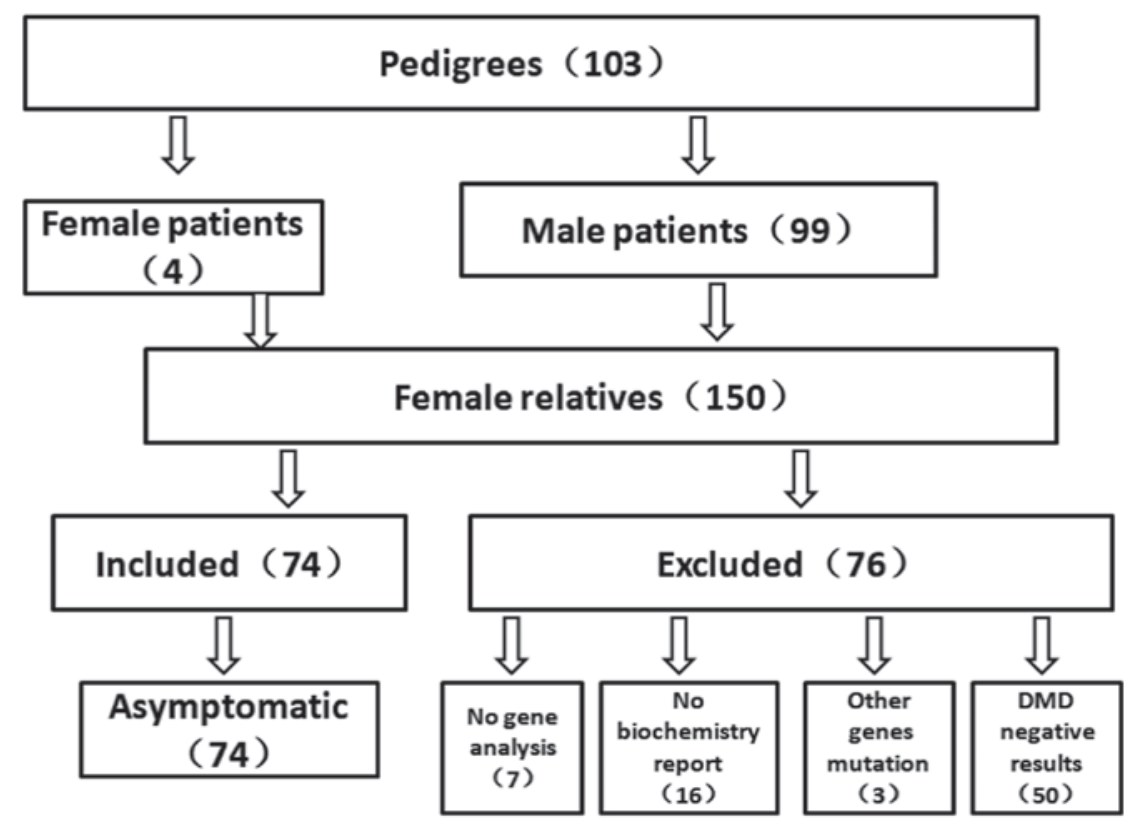

Figure 1. Flow diagram of the study.

identified using the GATK program (www.broadinstitute. org/gsa/wiki/index.php/Home_Page). The identified SNPs and InDels were annotated using the Exome-assistant program (http://122.228.158.106/exomeassistant).

Muscle pathology. Biopsy of the right gastrocnemius was performed in 2 female patients (both MCs) and 1 male DMD patient, and non-muscular dystrophy muscle tissue was utilized as a control. Each patient underwent open muscle biopsy from the right gastrocnemius under local anesthesia. Fresh specimens were fixed in $10 \%$ neutral buffered formalin and further processed into paraffin-embedded blocks. The morphology was observed under a microscope following hematoxylin and eosin staining. In addition, immunohistochemistry was used to evaluate dystrophin protein expression. Briefly, sections were incubated at $4^{\circ} \mathrm{C}$ overnight with a rabbit polyclonal antibody targeting dystrophin (cat. no. RB-9024; 1:100; Thermo Fisher Scientific, Inc.), and then incubated at room temperature for $30 \mathrm{~min}$ with Supervision TM Universal (6) (Anti-Mouse/Rabbit) Detection Reagent (HRP) (cat. no. D-3004; Lab Vision Corporation, Fremont, CA, USA) conjugated to peroxidase in Tris-HCI buffer containing carrier protein and anti-microbial agent. The experiment was performed according to the manufacturer's protocol.

Statistical analysis. Statistical analysis was conducted using SPSS software (version 16.0; SPSS, Inc., Chicago, IL, USA). Independent-samples t-test was used to compare the difference in CK level between Group 1 (MCs) and Group 2 (asymptomatic female carriers with high CK levels). Pearson's correlation coefficient (r) was used to identify the probable correlation of CK level with the AST and ALT levels in female carriers. Receiver operating characteristic (ROC) curve analysis was used to distinguish the predicted value of $\mathrm{CK}$ in DMD/BMD carriers. $\mathrm{P}<0.05$ was considered to denote differences that were statistically significant.

\section{Results}

Participant characteristics. A total of 103 families from the south of China were recruited in the present study, including 4 MCs and 99 male DMD patients. Furthermore, 150 female relatives were collected. Of these 150 participants, 23 participants were excluded due to incomplete clinical information, and 3 participants were excluded due to testing positive for a mutation in other genes that resulted in muscular dystrophy disease, confirmed as LGMD by muscle disease panel analysis. A homozygous mutation c. $583 \mathrm{G}>\mathrm{A}$ in the SGCA gene (LGMD2D), compound heterozygous mutations c.620T $>C$ and c.823C $>\mathrm{T}$ in the FKRP gene (LGMD2I), and compound heterozygous mutations c.77848C $>\mathrm{T}$ and c.97099C $>\mathrm{T}$ in the TTN gene (LGMD2J) were detected in these 3 cases. A total of 50 participants were also excluded as their DMD genetic analysis results were negative. Ultimately, 78 females were enrolled into the present study. Among these, 4 cases were MCs and 74 cases were asymptomatic female carriers, including 2 cases who were carriers of suspected germline mosaicism with no mutation in the dystrophin gene (Fig. 1).

Clinical symptoms. The present study included 4 symptomatic female carriers. Two of these MCs were admitted to the hospital due to elevated CK levels. All exhibited a variety of symptoms, including falls, waddling gait and tiredness (Table I).

\section{Laboratory examination}

Variation of $C K$ level. The $4 \mathrm{MCs}$ exhibited an increased CK level (100\%), and the mean CK level was 4,584.75 U/1. Among the 74 asymptomatic carriers, 47 cases had high $\mathrm{CK}$ levels, including 45 first-degree relatives and 2 sec-degree relatives of DMD/BMD probands (Fig. 2), with a mean $\mathrm{CK}$ level of 987.8 U/1 (range, 180-7,012 U/1). The ROC curve analysis revealed that the $\mathrm{CK}$ level was an excellent predictor for distinguishing DMD/BMD carriers. The area under the 


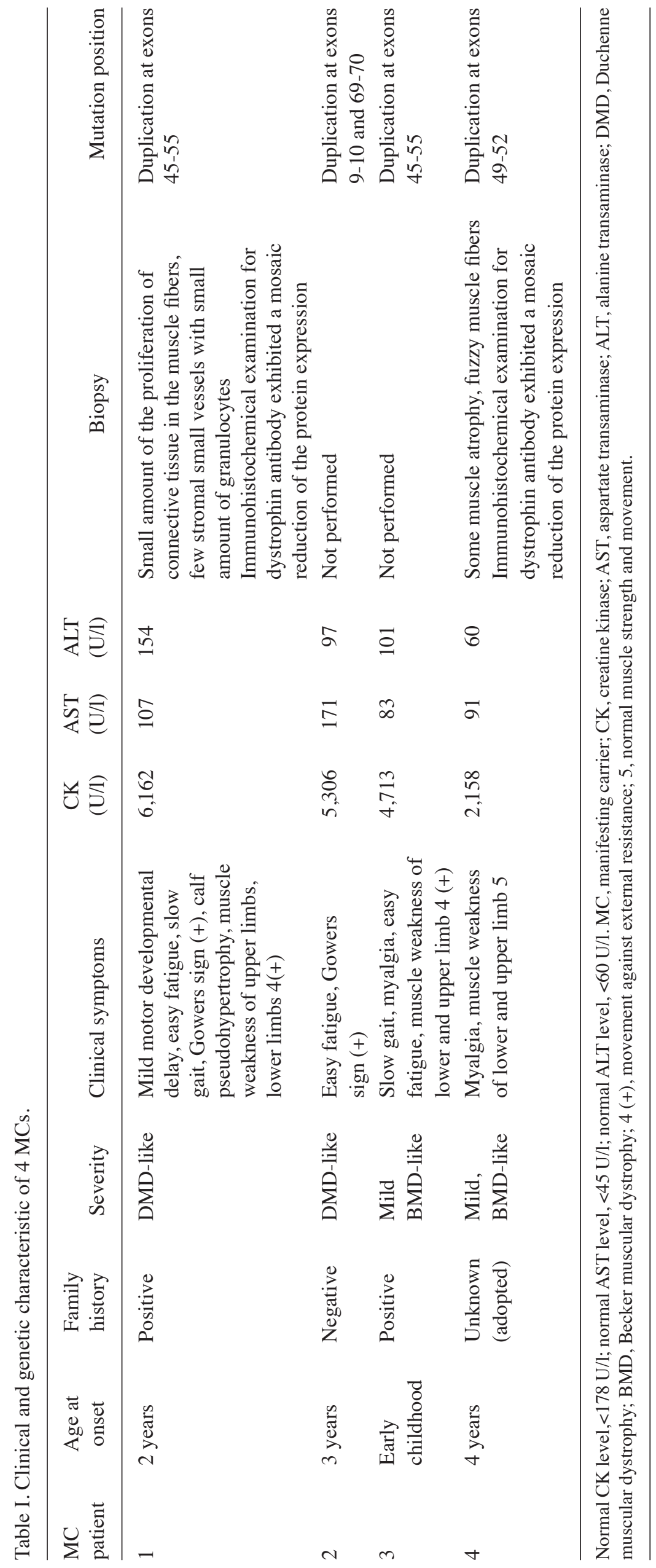


Table II. CK level of all female carriers included in the study.

\begin{tabular}{lcl}
\hline Group & No. $(\mathrm{n}=78)$ & \multicolumn{1}{c}{ CK level (U/l) } \\
\hline Group 1 & 4 & $6162,5306,4713,2158$ \\
Group 2 & 47 & $7012,4142,2724,2078,2065,1647,1516,1454,1419,1390,1233,1183,1169,1095,967,944$, \\
& & $920,855,794,759,758,655,633,582,550,525,515,500,494,484,460,434,431,415,372,355$, \\
& & $335,325,322,322,318,288,242,197,186,183,180$ \\
Group 3 & 27 & $174,167,157,154,147,135,128,128,128,118,110,106,102,97,96,90,84,83,81,76,73,72$, \\
& & $71,69,66,61,24$
\end{tabular}

Group 1 represents the manifesting carriers; Group 2 represents asymptomatic female carriers with a high CK level; and Group 3 represents asymptomatic female carriers with a normal CK level. CK, creatine kinase.

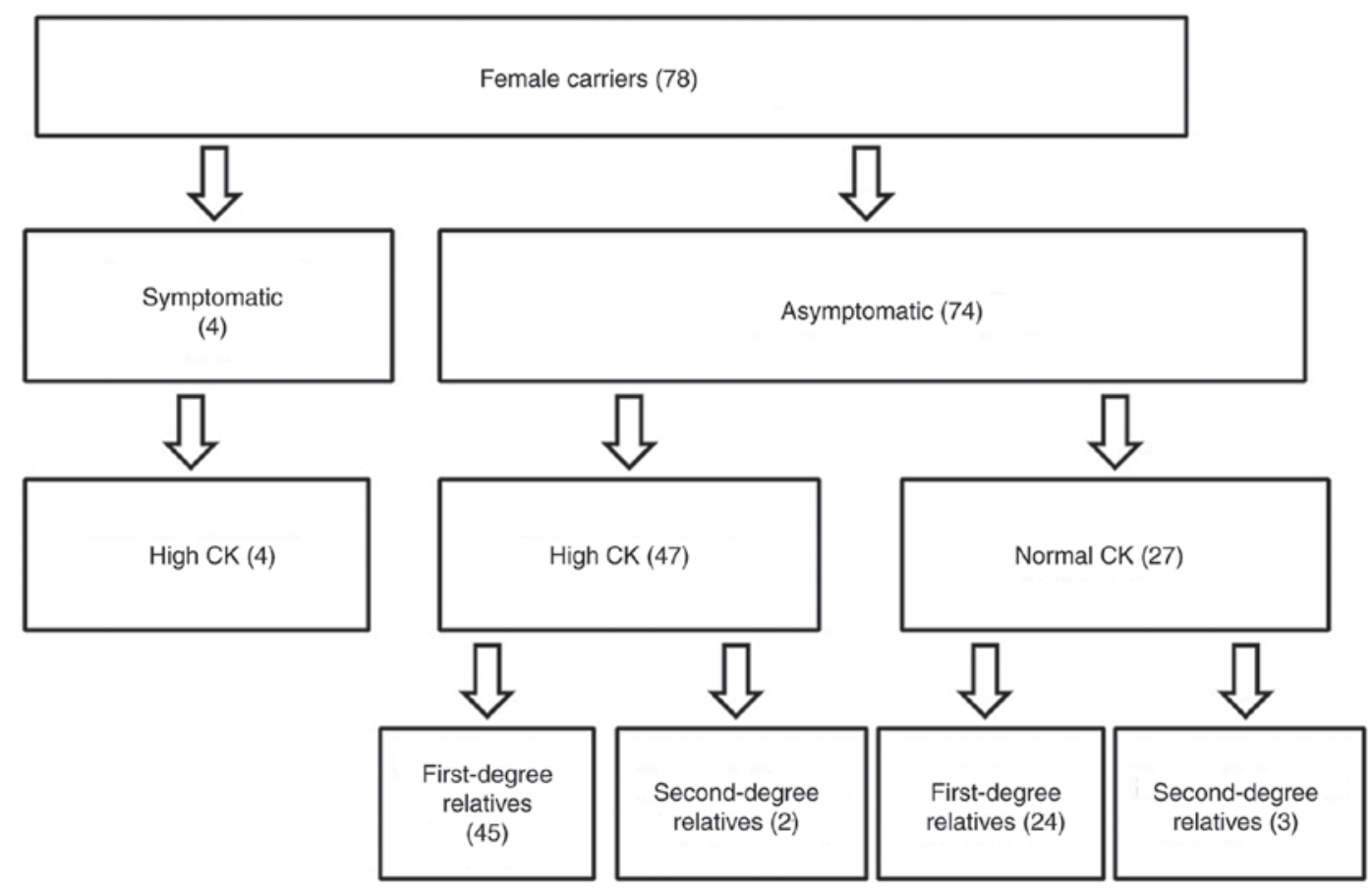

Figure 2. Population distribution and consanguinity of female carriers with different level of CK level. CK, creatine kinase; DMD, Duchenne muscular dystrophy; NGS, next-generation sequencing; MLPA, multiplex ligation-dependent probe amplification.

ROC curve was $0.822(\mathrm{P}<0.001)$, the sensitivity was $65.38 \%$ and the specificity was $92.1 \%$ (Fig. 3). Furthermore, a total of 27 asymptomatic female carriers had normal CK levels, accounting for 24 first-degree relatives and 3 sec-degree relatives (Fig. 2). The mean $\mathrm{CK}$ level of these subjects was 103.59 U/1 (range, 24-174 U/1; Table II). Female carriers were then divided into 3 groups according to their $\mathrm{CK}$ level: Group 1, MCs; Group 2, asymptomatic female carriers with high CK levels; and Group 3, asymptomatic female carriers with normal CK levels (Table II). Independent-samples t-test was used to compare the difference in CK level between Group 1 and Group 2, indicating that the CK level of MCs was significant higher in comparison with that of asymptomatic female carriers with high $\mathrm{CK}(\mathrm{P}<0.001)$.

Correlation of CK with AST and ALT levels. Among the 4 symptomatic and 74 asymptomatic female carriers, 61 cases exhibited normal ALT and AST levels, whereas 17 cases had elevated ALT and AST levels. The mean ALT level was $33.56 \mathrm{U} / 1$, with a minimum of $9 \mathrm{U} / 1$ and a maximum of $154 \mathrm{U} / 1$. Similarly, the mean AST level was $38.20 \mathrm{U} / 1$, with a minimum of $14 \mathrm{U} / 1$ and a maximum of $171 \mathrm{U} / 1$. Further analysis indicated that the variation in ALT and CK levels exhibited a positive correlation ( $\mathrm{r}=0.753, \mathrm{P}<0.05$; Fig. 4 ), while a positive correlation was also observed between AST and CK levels ( $\mathrm{r}=0.791$, $\mathrm{P}<0.05$; Fig. 5). AST exhibited a stronger correlation with $\mathrm{CK}$ levels as compared with that of ALT and CK levels.

Molecular characteristics of female carriers. In the present study, 4 MCs were detected by MLPA analysis of the dystrophin gene exons. All of the MCs exhibited duplication mutations, and duplications of exons 9-10, 69-70, 49-52 and 45-55 were identified. The other 74 asymptomatic carriers were analyzed by MLPA combined with Sanger sequencing. Among these, $31(41.89 \%)$ cases carried deletion mutations, $33(44.59 \%)$ cases exhibited point mutations, and only 10 cases $(13.51 \%)$ 


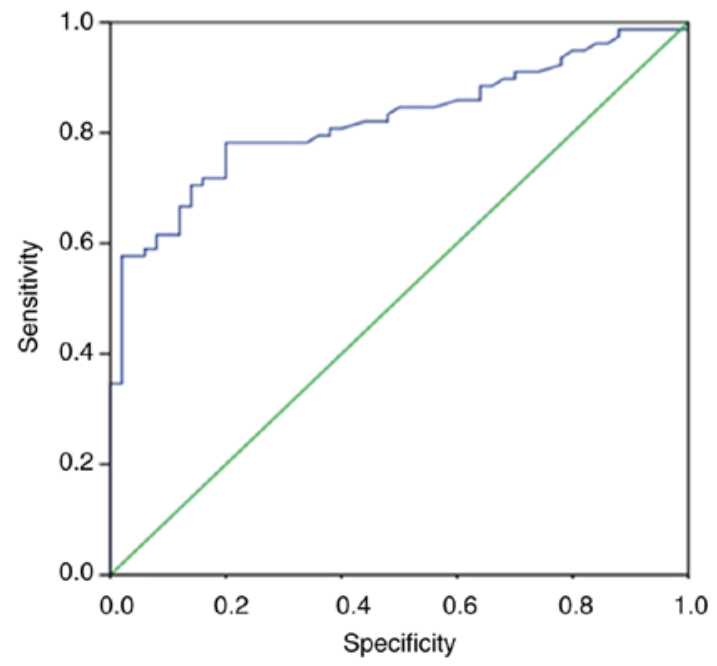

Figure 3. Receiver operating characteristic curve for CK level to classify Duchenne/Becker muscular dystrophy carriers. The area under the curve was 0.822 (ideal value, 1.00). This indicated an excellent diagnostic accuracy, which suggests that $\mathrm{CK}$ level has high specificity and sensitivity for the diagnosis of female carriers. CK, creatine kinase.

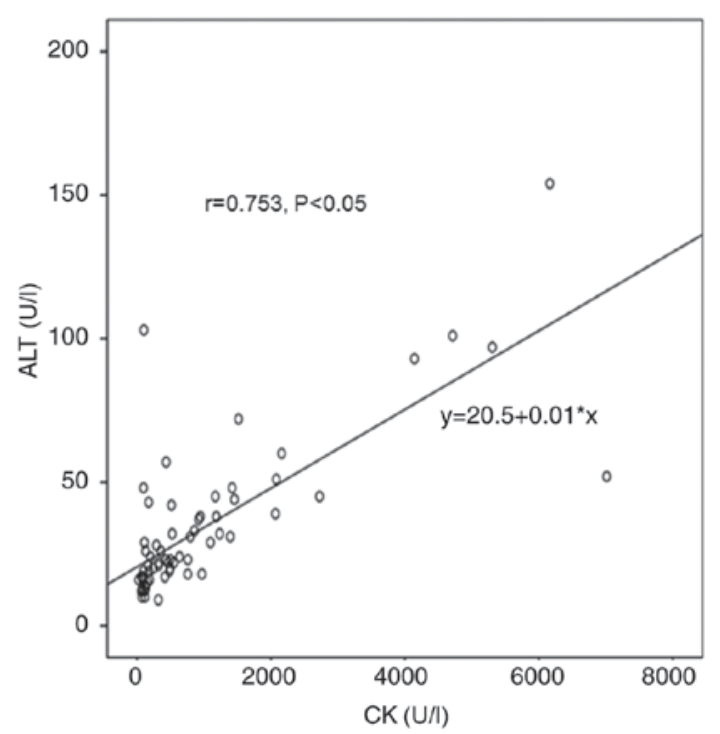

Figure 4. Serum ALT and CK levels exhibit a linear correlation ( $r=0.753$, $\mathrm{P}<0.05)$ in female carriers. ALT, alanine transaminase; CK, creatine kinase.

exhibited duplication mutations. In addition, 2 of the asymptomatic females were carriers of suspected germline mosaicism without mutations in dystrophin gene, although their daughters had the same mutations as the probands. Overall, 34 different mutations were characterized in the present study. The deletion breakpoints were mainly clustered at exons $45-55$, followed by exons 3-16 (Fig. 6A), whereas duplication breakpoints were clustered at the $3^{\prime}$ end of the dystrophin gene (Fig. 6B). The point mutation detection rate and distribution of mutations according to type, including 22 nonsense, 4 frame shift and 7 splice site mutations, are shown in Table III.

In addition, it was observed that $31(36 \%)$ mothers were negative for dystrophin gene analysis, and the associated probands had possible de novo mutations. A total of 6 female relatives exhibited negative genetic results by MLPA and NGS,

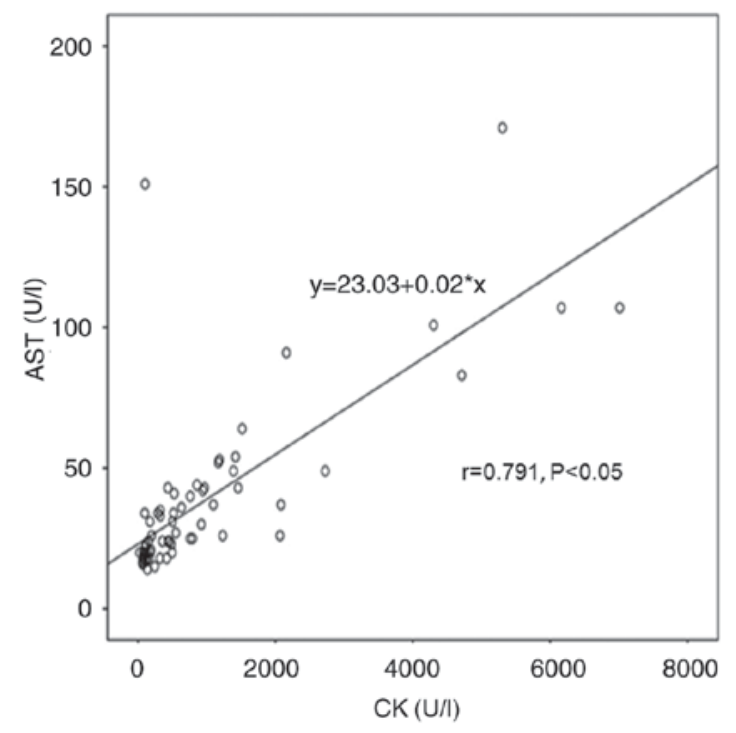

Figure 5. Serum AST and CK levels exhibit a linear correlation $(r=0.791$, $\mathrm{P}<0.05$ ) in female carriers. AST, aspartate transaminase; $\mathrm{CK}$, creatine kinase.
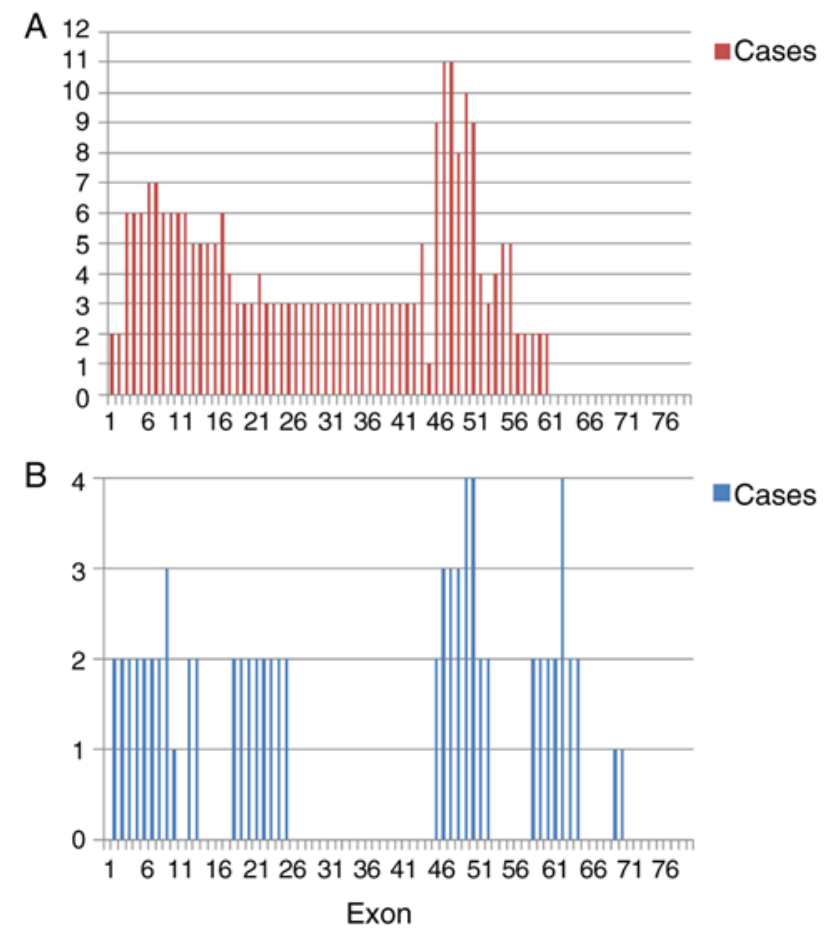

Figure 6. Frequency of deletion and duplication for all 79 exons of the dystrophin gene was analyzed by multiplex ligation-dependent probe amplification. In total, 31 cases carried deletion mutations, while 10 carried duplication mutations. (A) Deletion mutations were detected in the majority of exons, with the exception of exons 61-79, and the deletion hotspot regions were exons 45-55, followed by exons 3-16. (B) Duplication mutations were clustered at the 3 ' end of the dystrophin gene.

however, their CK levels were abnormally high (Table IV). These cases were first degree relatives of the probands, and a muscle disease diagnosis could not be made by muscle panel analysis. Therefore, the possibility that these subjects were DMD carriers cannot be excluded, particularly given the possibility of somatic mosaicism. Certainly, other factors that would cause high CK levels should be considered. 
Table III. Point mutations of female carriers, analyzed by Sanger sequencing.

\begin{tabular}{|c|c|c|c|c|}
\hline Patient & Base change & Effect & Exon ID & Mutation type \\
\hline 1 & c. $2605 \mathrm{C}>\mathrm{T}$ & P.Gln869Ter & Exon 20 & Nonsense \\
\hline 2 & c. $6318 \mathrm{G}>\mathrm{A}$ & p.Trp2106Ter & Exon 44 & Nonsense \\
\hline 3 & c.1860_1861delGT & p.Leu620LeufsTer14 & Exon 16 & Frameshift \\
\hline 4 & c. $8027+1 G>A$ & - & Intron 54 & Splicing \\
\hline 5 & c.8087 delT & p.Leu2696ArgfsTer30 & Exon 55 & Frameshift \\
\hline 6 & c. $6292 \mathrm{C}>\mathrm{T}$ & p.Arg2098Ter & Exon 44 & Nonsense \\
\hline 7 & c. $5488 \mathrm{~A}>\mathrm{T}$ & p.Arg1830Ter & Exon 39 & Nonsense \\
\hline 8 & c. $1332-9 A>G$ & - & Intron 11 & Splicing \\
\hline 9 & c. $7657 \mathrm{C}>\mathrm{T}$ & p.Arg2553Ter & Exon 52 & Nonsense \\
\hline 10 & c. $133 \mathrm{C}>\mathrm{T}$ & p.Gln45Ter & Exon 3 & Nonsense \\
\hline 11 & c. $8010 \mathrm{G}>\mathrm{A}$ & p.Trp2670Ter & Exon 54 & Nonsense \\
\hline 12 & c.10498_10499delAG & p.Ser3500Ter & Exon 74 & Frameshift \\
\hline 13 & c. $10108 \mathrm{C}>\mathrm{T}$ & p.Arg3370Ter & Exon 70 & Nonsense \\
\hline 14 & c. $3151 \mathrm{C}>\mathrm{T}$ & p.Arg1051Ter & Exon 23 & Nonsense \\
\hline 15 & c. $10223+1 \mathrm{G}>\mathrm{A}$ & - & Intron 69 & Splicing \\
\hline 16 & c. $.5488 \mathrm{~A}>\mathrm{T}$ & pArg 1830Ter & Exon 39 & Nonsense \\
\hline 17 & c. $3982 \mathrm{C}>\mathrm{T}$ & p.Gln1328Ter & Exon 29 & Nonsense \\
\hline 18 & c. $490 \mathrm{~A}>\mathrm{T}$ & p.K164X & Exon 6 & Nonsense \\
\hline 19 & c. $10108 \mathrm{C}>\mathrm{T}$ & p.Arg3370Ter & Exon 70 & Nonsense \\
\hline 20 & c. $3982 \mathrm{C}>\mathrm{T}$ & p.Gln1328Ter & Exon 29 & Nonsense \\
\hline 21 & c. $3721 \mathrm{G}>\mathrm{T}$ & p.Glu1241Ter & Exon 27 & Nonsense \\
\hline 22 & c. $10171 \mathrm{C}>\mathrm{T}$ & p.Arg3391Ter & Exon 70 & Nonsense \\
\hline 23 & c. $10171 \mathrm{C}>\mathrm{T}$ & p.Arg3391Ter & Exon 70 & Nonsense \\
\hline 24 & c. $1332-8 \mathrm{~A}>\mathrm{G}$ & - & Intron 11 & Splicing \\
\hline 25 & c. $3603+1 \mathrm{G}>\mathrm{A}$ & - & Intron 26 & Splicing \\
\hline 26 & c. $1800-1 \mathrm{G}>\mathrm{A}$ & - & Intron 14 & Splicing \\
\hline 27 & c. $462+1 \mathrm{G}>\mathrm{T}$ & - & Intron 6 & Splicing \\
\hline 28 & c. $133 \mathrm{C}>\mathrm{T}$ & p.Gln45Ter & Exon 3 & Nonsense \\
\hline 29 & c. $691 \mathrm{~T}>\mathrm{A}$ & p.Tyr231Asn & Exon 8 & Frameshift \\
\hline 30 & c. $5488 \mathrm{~A}>\mathrm{T}$ & pArg1830Ter & Exon 39 & Nonsense \\
\hline 31 & c. $5488 \mathrm{~A}>\mathrm{T}$ & pArg 1830Ter & Exon 39 & Nonsense \\
\hline 32 & c. $10171 \mathrm{C}>\mathrm{T}$ & p.Arg3391Ter & Exon 70 & Nonsense \\
\hline 33 & c. $3151 \mathrm{C}>\mathrm{T}$ & p.Arg1051Ter & Exon 23 & Nonsense \\
\hline
\end{tabular}

Table IV. CK level of female relatives with high CK level and negative genetic results.

\begin{tabular}{|c|c|c|c|c|}
\hline Female relative & CK (U/1) & $\operatorname{AST}(\mathrm{U} / \mathrm{l})$ & $\operatorname{ALT}(\mathrm{U} / \mathrm{l})$ & Mutation of proband \\
\hline 1 & 297 & 24 & 18 & DEL EX46-59 \\
\hline 2 & 194 & 14 & 19 & DEL EX46-48 \\
\hline 3 & 632 & 19 & 18 & DEL EX45-55 \\
\hline 4 & 257 & 20 & 16 & DEL EX50-54 \\
\hline 5 & 191 & 13 & 19 & DEL EX04-11 \\
\hline 6 & 311 & 38 & 43 & DEL EX51 \\
\hline
\end{tabular}

Normal CK level, <178 U/l; normal AST level, <45 U/l; normal ALT level, <60 U. CK, creatine kinase; AST, aspartate transaminase; ALT, alanine transaminase.

Muscle biopsy results. Among the 4 symptomatic carriers, The proliferation of connective tissue in the muscle fibers 2 patients were subjected to muscle biopsy (Fig. 7A and B). was low, and individual interstitial small vessels cut through 
A

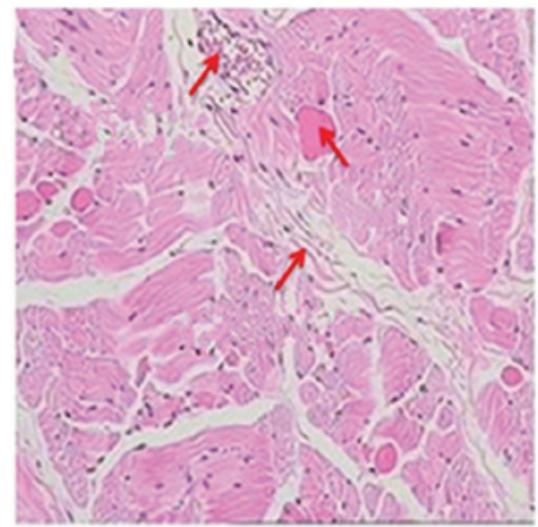

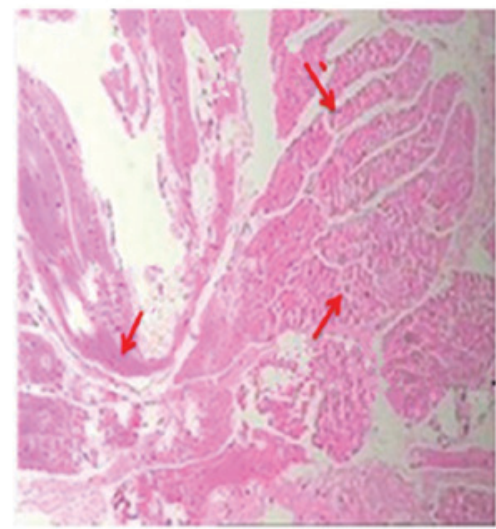

D

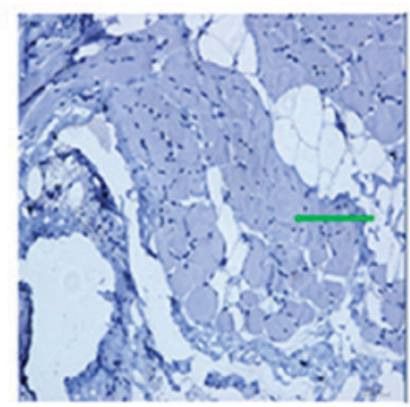

Figure 7. Sections with HE staining and immunolabeling with dystrophin antibodies of patient 1 (magnification, x100). (A) HE staining of muscle biopsies of patient 1, exhibiting a small amount of connective tissue proliferation in the muscle fibers, few stromal small vessels with a small amount of granulocytes and lymphocytes infiltration. Dystrophin expression in the biopsies obtained from a (B) control, (C) female MC (patient 1) and (D) male DMD patient. The control section presented a normal expression of dystrophin, with a consistent and uniform brown muscle fiber membrane. The MC (patient 1) exhibited random presence of normal and dystrophin-deficient fibers, indicating a mosaic expression of dystrophin. By contrast, the male DMD patient exhibited almost no expression of dystrophin in the muscle fiber membrane. The red arrow indicates expression of dystrophin in brown muscle fiber membrane, and the green arrow indicates absence of dystrophin expression. HE, hematoxylin-eosin; MC, manifesting carrier; DMD, Duchenne muscular dystrophy.

only a few granulocytes and lymphocytes. 'Muscle fiber necrosis was observed and regeneration was not evident, except when combined with clinical myositis. The immune positivity of spectrin in all biopsies indicated well-preserved sarcolemmal integrity, ensuring that false negative results for other sarcolemmal membrane-associated proteins could be ruled out. Furthermore, immunohistochemical examination with monoclonal antibodies against dystrophin indicated a mosaic reduction of the protein expression in these MCs. The control biopsy presented a normal expression of dystrophin, with a consistent and uniform brown muscle fiber membrane. By contrast, in male DMD patients, almost no dystrophin expression was observed in the muscle fiber membrane (Fig. 7C-E).

\section{Discussion}

Dystrophinopathy is the most common muscular dystrophy and involves X-linked recessive inheritance, while MCs are rare. In the present study, $4 \mathrm{MC}$ females (4/78 participants; $4.49 \%$ ) were reported, who presented with muscle weakness and elevated CK levels. Duplication mutations in dystrophin gene were detected in all of the MCs. It has previously been reported that $2.5-10 \%$ carriers experience symptoms ranging from mild muscle weakness to rapidly progressive DMD-like muscular dystrophy (5), which is consistent with the results of the present study. Several disease-causing mechanisms have been implicated in DMD/BMD MCs; however, the most frequently reported mechanism to provoke symptoms in $\mathrm{DMD} / \mathrm{BMD}$ carriers is skewed $\mathrm{X}$-inactivation (7). In $\mathrm{X}$-linked dominant diseases, $\mathrm{X}$ inactivation can influence the severity of the phenotype or the survival of females with heterozygous lethal mutations. Our next step will be relevant to the $\mathrm{X}$ inactivation analysis of these carriers to clarify its pathogenesis.

The phenotypes of MCs of DMD/BMD are similar to LGMD and are, therefore, likely to be misdiagnosed with LGMD. MCs with motor weaknesses should be distinguished from other myodystrophies. As many as $17 \%$ of dystrophinopathies are misdiagnosed as LGMD (8). In the present study, 3 cases among the 78 cases exhibited progressive motor weaknesses similar to DMD and elevated CK levels. A subsequent muscle disease panel analysis revealed their diagnosis as LGMD, which was confirmed by muscle biopsy and immunostaining. In addition to weakness symptoms, an MC with motor developmental delay was detected, but without any other intellectual or cognitive disorders or mental illnesses. These findings are in accordance with those reported by Seeman et al (9) in 2010. More recently, the incidence of mental retardation in MCs was found to be as high as $7 \%$ (10). However, all 4 female symptomatic carriers in the present study did not exhibit evidence of mental retardation.

As CK is a component of the muscle fiber cytosol, its serum activity is considered to be positively correlated with 
progressing muscle damage. An elevated CK level is one of the characteristic features of $\mathrm{DMD} / \mathrm{BMD}$, particularly when screening neonatal carriers. To the best of our knowledge, the present study is the first to use a ROC curve to analyze the diagnostic sensitivity of CK level in DMD/BMD, and the results suggested that the $\mathrm{CK}$ level has high specificity and sensitivity for the diagnosis of female carriers. For female relatives with negative genetic tests, particularly those with $\mathrm{CK}$ values up to 1.5-3 times the normal upper limit, muscle biopsy and immunohistochemical analyses are recommended to evaluate dystrophin protein expression to confirm the status of probable carriers. As previous studies have suggested, chronic persistent $\mathrm{CK}$ elevation indicates the possibility of neuromuscular disease (11). Wang et al (12) reported that among a group of 201 female relatives, 97 of the 98 first-degree relatives with high levels of CK were DMD or BMD carriers, while 31 of the remaining 103 subjects exhibiting normal CK levels were diagnosed as carriers. Therefore, the possibility that female relatives with normal levels of $\mathrm{CK}$ could be carriers cannot be excluded. In the present study, it was also observed that CK levels were positively correlated with the levels of ALT and AST. Elevated ALT/AST may be the presenting sign of muscle disease in children, which provides an opportunity for early diagnosis. For patients with unexplained long-lasting hypertransaminasemia,serum $\mathrm{CK}$ activity should be measured and muscle biopsy should be conducted at an early stage for correct diagnosis of muscular dystrophy. The sensitivity of ALT and AST was observed to be $21.79 \%$ in the present study, which is significantly lower compared with that for the $\mathrm{CK}$ level. Therefore, the CK level is a better predictor of DMD/BMD carriers, and female relatives with elevated ALT/AST should also be screened for serum CK activity to identify potential carriers. While a total of 6 female relatives in the present study had a high CK level, they did not present any mutations in the dystrophin gene. Since these patients did not agree to undergo muscle biopsy, they cannot be excluded as possible carriers.

The limitations of MLPA and NGS analyses can lead to false negatives, while germline mosaicism carriers would also show a negative result in genetic tests. Luce et al (13) reported three recombination events and suggested that germline mosaicism had occurred in these families. In addition, high CK levels may be due to other non-neuromuscular factors, such as physical activity or muscle trauma (14), and further study would help to exclude the possibility of myopathy. Currently, muscle biopsy immunostaining has become important for the diagnosis of myopathy. In the present study, the muscle biopsy immunostaining results of two symptomatic carriers exhibited mosaic distribution. Approximately $20 \%$ of carriers had abnormal dystrophin immunostaining on muscle biopsy, with a mosaic pattern of dystrophin-positive and dystrophin-negative fibers present (15). When the gene mutation analysis is negative, muscle biopsy with immunohistochemical staining would be useful to assist in the further diagnosis of carriers.

In the present study, 74 asymptomatic carriers were detected by MLPA combined with Sanger sequencing. Among these, $41.89 \%$ of cases involved deletion mutations, $44.59 \%$ of cases exhibited point mutations in exons and $13.51 \%$ exhibited duplication mutations. These results are similar to those of a previous study (16). Additionally, all of 4 MCs carried frameshift and duplication mutations. By contrast, point mutations and deletions were more common in female asymptomatic carriers, as previously reported in the literature (17). More frameshift and duplication mutations were observed in MCs in the present study, which may be due to the small sample size. Therefore, larger samples should be used in future studies. Large segmental deletions and duplications in female carriers of dystrophin gene mutations involved the majority of the exons. Deletion breakpoints were mainly clustered at exons $45-55$, followed by exons $3-16$, which was similar to the results previously reported in the literature (4). However, in the present study, duplications were concentrated in the $3^{\prime}$ end of the DMD gene, in contrast with previously reported DMD gene mutation hot spots at the 5' end (17). An earlier study analyzed 92 suspected DMD/BMD male patients, and reported that the deletion and duplication hot spots in the dystrophin gene were the same as those of female carriers in the present study (18). The discrepancy between the results of the present and previous studies may be due to the small sample we collected.

According to the literature, approximately two-thirds of the genetic mutations in male DMD/BMD patients were inherited from their carrier mothers, while more than one-third were de novo mutations (19). In the present study, 31 (36\%) mothers did not exhibit a mutation in the dystrophin gene, as determinedby MLPA and Sanger sequencing analysis. This indicated that these probands may present de novo mutations. Furthermore, 2 cases of germline mutation female carriers who were the mothers of the 2 probands were identified They did not exhibit a mutation in the dystrophin gene by MLPA and NGS analyses, and had a normal CK level. However, their daughters presented the same mutation as the probands, although this mutation was absent in the fathers of these cases. This evidence suggests that these mothers are highly likely to have germline mosaicism. Thus, mothers with a DMD/BMD child should be aware of the possibility that they are DMD/BMD carriers, particularly those who show an elevated CK level, even when no mutation in the dystrophin gene is detected by genetic analysis. A number of researchers recommend that mothers who have a DMD child should obtain prenatal diagnosis again when pregnant. In order to prevent DMD/BMD, attention must be paid to dystrophinopathy carrier screening, genetic counseling and prenatal diagnosis.

In conclusion, measurement of the CK level is a non-invasive, easy and excellent screening method for DMD/BMD carriers, and this level was observed to be strongly correlated with ALT and AST. The present study reported that MLPA for exons of the dystrophin gene, along with a muscle disease panel analysis, was effective for the diagnosis of MCs, while MLPA combined with Sanger sequencing determined carrier status. However, a number of carriers remained that could not be confirmed by this strategy. DMD/BMD female relatives with negative genetic testing results, particularly those with high CK levels, are recommended to undergo prenatal diagnosis. Furthermore, female relatives with negative genetic tests and persistent high CK levels should be recommended for muscle biopsy and immunohistochemical analysis to evaluate dystrophin protein expression in order to confirm the status of probable carriers. 


\section{Acknowledgements}

Not applicable.

\section{Funding}

This study was supported by the Chinese Natural Science Foundation (grant no. 81760215), the First Affiliated Hospital of Guangxi Medical University starting fund for study abroad returnees (grant no. 2010001), and the Natural Science Foundation of Guangxi Province (grant no. 03201216025D).

\section{Availability of data and materials}

The datasets used and/or analyzed during the current study are available from the corresponding author on reasonable request.

\section{Authors' contributions}

DL conducted the study and analyzed the genetic test results. JZho and YX summarized the clinical information, analyzed the genetic test results and drafted the manuscript. VB helped to summarize the clinical information and analyze the genetic test results. GC analyzed the pathological biopsy results. YD performed the pathological biopsy. HL and JZha obtained the clinical information. All authors read and approved the final manuscript.

\section{Ethics approval and consent to participate}

All procedures performed in this study involving human participants were in accordance with the ethical standards of the institutional and national research committee. Informed consent was obtained from all participants included in the study.

\section{Patient consent for publication}

Consent for publication was obtained from all participants included in the study.

\section{Competing interests}

The authors declare that they have no competing interests.

\section{References}

1. Bushby K, Finkel R, Birnkrant DJ, Case LE, Clemens PR, Cripe L, Kaul A, Kinnett K, McDonald C, Pandya S, et al: Diagnosis and management of Duchenne muscular dystrophy, part 1: Diagnosis, and pharmacological and psychosocial management. Lancet Neurol 9: 77-93, 2010

2. Ryder S, Leadley RM, Armstrong N, Westwood M, de Kock S, Butt T, Jain M and Kleijnen J: The burden, epidemiology, costs and treatment for Duchenne muscular dystrophy: An evidence review. Orphanet J Rare Dis 12: 79, 2017.

3. Magri F, Govoni A, D'Angelo MG, Del Bo R, Ghezzi S, Sandra G, Turconi AC, Sciacco M, Ciscato P, Bordoni A, et al: Genotype and phenotype characterization in a large dystrophinopathic cohort with extended follow-up. J Neurol 258: 1610-1623, 2011.
4. Lopez-Hernandez LB, Gomez-Diaz B, Luna-Angulo AB, Anaya-Segura $M$, Bunyan DJ, Zuniga-Guzman C, Escobar-Cedillo RE, Roque-Ramirez B, Ruano-Calderon LA, Rangel-Villalobos $\mathrm{H}$, et al: Comparison of mutation profiles in the Duchenne muscular dystrophy gene among populations: Implications for potential molecular therapies. Int J Mol Sci 16: 5334-5346, 2015

5. Taylor PJ, Maroulis S, Mullan GL, Pedersen RL, Baumli A, Elakis G, Piras S, Walsh C, Prosper-Gutierrez B De La Puente-Alonso F, et al: Measurement of the clinical utility of a combined mutation detection protocol in carriers of Duchenne and Becker muscular dystrophy. J Med Genet 44: 368-372, 2007.

6. Yang P, Du CW, Kwan M, Liang SX and Zhang GJ: The impact of p53 in predicting clinical outcome of breast cancer patients with visceral metastasis. Sci Rep 3: 2246, 2013.

7. Juan-Mateu J, Rodriguez MJ, Nascimento A, JiménezMallebrera C, González-Quereda L, Rivas E, Paradas C, Madruga M, Sanchez-Ayaso P, Jou C, et al: Prognostic value of $\mathrm{X}$-chromosome inactivation in symptomatic female carriers of dystrophinopathy. Orphanet J Rare Dis 7: 82, 2012.

8. Arikawa E, Hoffman EP, Kaido M, Nonaka I, Sugita H and Arahata K: The frequency of patients with dystrophin abnormalities in a limb-girdle patient population. Neurology 41: 1491-1496, 1991.

9. Seemann N, Campbell C, Hammond R and Prasad C: 9 year old girl with progressive weakness. Brain Pathol 20: 255-256, 2010.

10. Mercier S, Toutain A, Toussaint A, Raynaud M, de Barace C, Marcorelles P, Pasquier L, Blayau M, Espil C, Parent P, et al: Genetic and clinical specificity of 26 symptomatic carriers for dystrophinopathies at pediatric age. Eur J Hum Genet 21: 855-863, 2013.

11. Dabby R, Sadeh M, Herman O, Berger E, Watemberg N, Hayek S, Jossiphov J and Nevo Y: Asymptomatic or minimally symptomatic hyperCKemia: Histopathologic correlates. Isr Med Assoc J 8: 110-113, 2006.

12. Wang Q, Yang X, Yan Y, Song N, Lin C and Jin C: Duchenne or Becker muscular dystrophy: A clinical, genetic and immunohistochemical study in China. Neurol India 59: 797-802, 2011.

13. Luce LN, Ottaviani D, Ferrer M, Szijan I, Cotignola J and Giliberto F: Molecular diagnosis of dystrophinopathies using a multi-technique analysis algorithm. Muscle Nerve 49: 249-256, 2014.

14. Voermans NC, de Visser M, Wokke JH and Brusse E: Increased CK activity in serum without symptoms: Further investigations often unnecessary. Ned Tijdschr Geneeskd 157: A6315, 2013 (In Dutch).

15. Hoogerwaard EM, Ginjaar IB, Bakker E and de Visser M: Dystrophin analysis in carriers of Duchenne and Becker muscular dystrophy. Neurology 65: 1984-1986, 2005.

16. Soltanzadeh P, Friez MJ, Dunn D, von Niederhausern A, Gurvich OL, Swoboda KJ, Sampson JB, Pestronk A, Connolly AM, Florence JM, et al: Clinical and genetic characterization of manifesting carriers of $D M D$ mutations. Neuromuscul Disord 20: 499-504, 2010

17. Lee SH, Lee JH, Lee KA and Choi YC: Clinical and genetic characterization of female dystrophinopathy. J Clin Neurol 11: 248-251, 2015

18. Zhong J, Xu T, Chen G, Liao H, Zhang J and Lan D: Genetic analysis of the dystrophin gene in children with Duchenne and Becker muscular dystrophies. Muscle Nerve 56: 117-121, 2017.

19. Mukherjee M, Chaturvedi LS, Srivastava S, Mittal RD and Mittal B: De novo mutations in sporadic deletional Duchenne muscular dystrophy (DMD) cases. Exp Mol Med 35: 113-117, 2003.

This work is licensed under a Creative Commons Attribution-NonCommercial-NoDerivatives 4.0 International (CC BY-NC-ND 4.0) License. 